\title{
Adenosine Deaminases Acting on RNA Downregulate the Expression of Constitutive Androstane Receptor in the Human Liver-Derived Cells by Attenuating Splicing
}

\author{
Masataka Nakano, Tatsuki Fukami, and Miki Nakajima \\ Drug Metabolism and Toxicology, Faculty of Pharmaceutical Sciences and WPI Nano Life Science Institute, Kanazawa \\ University, Kakuma-machi, Kanazawa, Japan
}

Received May 18, 2019; accepted June 28, 2019

\begin{abstract}
Adenosine deaminases acting on RNA (ADARs) enzymescatalyzing adenosine-to-inosine RNA editing possibly modulates gene expression and function. In this study, we investigated whether ADARs regulate the expression of human constitutive androstane receptor (CAR), which controls the expression of various drug-metabolizing enzymes. CAR mRNA and protein levels in human hepatocellular carcinoma-derived HepG2 cells were increased by knockdown of ADAR1 and slightly increased by ADAR2, indicating that ADARs negatively regulate CAR expression. Increased luciferase activity of a reporter plasmid containing the CYP3A4 promoter region by phenobarbital was augmented by transfection of siRNA for ADAR1 (siADAR1) but not by siADAR2. In addition, the knockdown of ADAR1 resulted in the enhanced induction of CYP2B6 and CYP3A4 mRNA by 6(4-chlorophenyl)imidazo[2,1-b][1,3]thiazole-5-carbaldehyde O(3,4-dichlorobenzyl)oxime and phenobarbital, respectively. These results suggest that ADAR1-mediated downregulation of CAR affects its downstream cytochrome P450 expression.
\end{abstract}

When the transcription was inhibited by $\alpha$-amanitin, the degradation of CAR mRNA was attenuated by knockdown of ADAR1, suggesting that the increase in CAR mRNA level by ADAR1 knockdown is a post-transcriptional event. Finally, we found that ADAR1 knockdown promotes the splicing of CAR as a mechanism of the increased expression of CAR by ADAR1 knockdown. In conclusion, this study revealed that ADAR1 plays a role in modulating xenobiotic metabolism potency via regulation of CAR.

\section{SIGNIFICANCE STATEMENT}

This study revealed that adenosine deaminase acting on RNA 1 (ADAR1) and ADAR2, which catalyze adenosine-to-inosine RNA editing, downregulate the expression of constitutive androstane receptor (CAR) in human liver-derived cells by attenuating splicing. The downregulation of CAR by ADARs affected its downstream cytochrome P450 expression. ADARs would play a role in modulating xenobiotic metabolism potency via regulation of CAR.

\section{Introduction}

Understanding the regulatory mechanisms for drug metabolism-related genes is required for the practice of personalized or precision medicine, as well as for promoting efficient drug development. Regulation of gene expression is a multiple-layered process occurring at the levels of transcription, post-transcription, translation, and post-translation. Conventional studies on regulatory mechanisms for drugmetabolizing enzymes have focused primarily on transcriptional regulation. In addition, there is growing evidence showing a great impact of post-transcriptional regulation.

The authors' studies were supported by a Grant-in-Aid for Scientific Research (B) [18H02573] and Grant-in-Aid for Early Career Scientists [18K14901] from the Japan Society for the Promotion of Science, and World Premier International Research Center Initiative, Ministry of Education, Culture, Sports, Science and Technology, Tokyo, Japan.

https://doi.org/10.1124/jpet.119.260109.
As one of the post-transcriptional regulations, eukaryotic RNA transcripts are subjected to adenosine-to-inosine (A-to-I) RNA editing. A-to-I RNA editing is the most frequent nucleotide conversion type in mammals (Slotkin and Nishikura, 2013; Nishikura, 2016). During the past 10 years, the progress of next-generation sequencing technologies has enabled the high-throughput identification of 4.5 million A-to-I RNA editing sites (Picardi et al., 2017). A-to-I RNA editing is catalyzed by adenosine deaminase acting on RNA (ADAR) enzymes. There are two functional members of the ADAR family in humans: ADAR1 and ADAR2. These enzymes have a deaminase domain and double-stranded RNA (dsRNA) binding domains (Kim et al., 1994; Gerber et al., 1997); therefore, they convert adenosines in dsRNA structures into inosines by hydrolytic deamination. Inosine forms base pairs with cytidine as if it was guanosine. Thus, the nucleotide conversion in the coding region possibly changes the amino acid sequence. However, most editing events occur in noncoding regions, such as the introns and untranslated region

ABBREVIATIONS: ADAR, adenosine deaminase acting on RNA AS antisense; $A$ to I, adenosine to inosine; CAR, constitutive androstane receptor; CITCO, 6-(4-chlorophenyl)imidazo[2,1-b][1,3]thiazole-5-carbaldehyde O-(3,4-dichlorobenzyl)oxime; dsRNA, double-stranded RNA; ex, exon; GAPDH, glyceraldehyde-3-phosphate dehydrogenase; P450, cytochrome P450; PB, phenobarbital; PCR, polymerase chain reaction; PTC, premature translation-termination codon S Sense; siADAR, siRNA for human ADAR; siControl, siRNA for negative control; siRNA, small interfering RNA; UTR, untranslated region; RT-PCR, reverse-transcription PCR. 
(UTR) (Peng et al., 2012). Editing in the intron has potential to affect splicing (Samuel, 2019). Since the $3^{\prime}$-UTR is the main target of microRNAs, A-to-I RNA editing events in the $3^{\prime}$-UTR possibly create or destroy the binding site, resulting in the changes in mRNA stability and translational efficiency (Wang and Liang, 2018). Through these processes, RNA editing makes great impacts on gene expression and function of the encoded proteins.

Previously, we found that there is a large interindividual variability (220-fold) in ADAR1 protein levels in human livers (Nakano et al., 2016), which is the main tissue of drug metabolism. This fact prompted us to surmise a possibility that interindividual differences in ADAR expression and RNA editing levels may affect the drugmetabolism potencies (Nakano and Nakajima, 2018). More recently, we experimentally proved that ADARs modulate the expression of cytochrome P450 (P450) isoforms in differentiated HepaRG cells (Nozaki et al., 2019). Multiple human P450 isoforms, such as CYP2B6, CYP2C9, and CYP3A4, are transcriptionally regulated by constitutive androstane receptor (CAR, NR1I3), which is a member of the nuclear receptor superfamily and a crucial regulator of drug metabolism and elimination. CAR is activated by some synthetic compounds, drugs, or natural products (Chang and Waxman, 2006) and induces expression of UDP-glucuronosyltransferases, sulfotransferases, and drug transporters as well as P450s (Negishi and Honkakoski, 2000; Moore et al., 2006). Thus, CAR is recognized as a xenosensor for the detoxification of foreign compounds and a cause of interindividual difference in drug metabolism. Clarification of the regulation mechanisms of CAR expression would lead to understanding of the interindividual difference in drug metabolism potency. In the present study, we sought to expand the knowledge on ADAR-dependent regulation of pharmacokinetics, focusing on CAR.

\section{Materials and Methods}

Chemicals and Reagents. Phenobarbital (PB) was purchased from Wako Pure Chemical (Osaka, Japan). 6-(4-Chlorophenyl)imidazo [2,1-b][1,3]thiazole-5-carbaldehyde $O$-(3,4-dichlorobenzyl)oxime (CITCO) was purchased from Biomol Research Laboratories (Plymouth Meeting, PA). Lipofectamine RNAiMAX, Silencer Select small interfering RNA (siRNA) for human ADAR1 (s1007; siADAR1), human ADAR2 (s1010; siADAR2), and negative control \#1 (siControl) were obtained from Thermo Fisher Scientific (Waltham, MA). The pGL3-basic vector, pGL3-promoter vector, pGL4.17 vector, pNL1.1 vector, and Nano-Glo Dual-Luciferase Reporter Assay System were purchased from Promega (Madison, WI). RNAiso and random hexamers were from Takara (Shiga, Japan). ReverTra Ace was obtained from Toyobo (Osaka, Japan). Luna Universal qPCR Master Mix was from New England Biolabs (Ipswich, MA). All primers were commercially synthesized at IDT Technology (Coraville, IA). Rabbit anti-human CAR polyclonal antibody (AB10303) was from Chemicon International (Temecula, CA). Mouse anti-human ADAR1 monoclonal antibody (sc5579) and mouse anti-human ADAR2 monoclonal antibody (sc-73408) were purchased from Santa Cruz Biotechnology (Santa Cruz, CA). Rabbit anti-human glyceraldehyde-3-phosphate dehydrogenase (GAPDH) polyclonal antibody (IMG-5143A) was from IMGENIX (San Diego, CA). IRDye 680 goat anti-rabbit IgG and goat antimouse IgG were purchased from LI-COR Biosciences (Lincoln, NE).

Cell Cultures. Human hepatocellular carcinoma-derived HepG2 cells were obtained from the American Type Culture Collection (Manassas, VA). The cells were cultured in Dulbecco's modified Eagle's medium containing $10 \%$ fetal bovine serum (Invitrogen,
Carlsbad, CA) and $0.1 \mathrm{mM}$ nonessential amino acids (Invitrogen) in a $5 \% \mathrm{CO}_{2}$ atmosphere at $37^{\circ} \mathrm{C}$.

Transfection of siRNA into HepG2 Cells and Preparation of Cell Homogenates and Total RNA. HepG2 cells were seeded into six-well plates, and $5 \mathrm{nM}$ siRNA was transfected using Lipofectamine RNAiMAX. After 72 hours, the cells were collected, suspended in a small amount of Tris-glycerol-EDTA buffer [10 mM Tris-HCl, $20 \%$ glycerol, and $1 \mathrm{mM}$ EDTA ( $\mathrm{pH}$ 7.4)], and disrupted by freezethawing three times. Total RNA was prepared using RNAiso.

Evaluation of CYP2B6 and CYP3A4 mRNA Induction. HepG2 cells were seeded and transfected with siRNA as described earlier. After 48 hours, the cells were treated with $100 \mathrm{nM}$ CITCO or $1 \mathrm{mM}$ PB. After incubation for 24 hours, the cells were harvested, and total RNA was prepared.

Real-Time Reverse-Transcription Polymerase Chain Reaction. cDNA was synthesized from total RNA using ReverTra Ace. The used primers are shown in Table 1. A 1- $\mu$ l portion of the cDNA was added to a polymerase chain reaction (PCR) mixture containing 10 pmol of each primer and $10 \mu \mathrm{l}$ of the Luna Universal qPCR Master Mix in a final volume of $20 \mu \mathrm{l}$. Real-time PCR was performed using Mx3000P (Stratagene, La Jolla, CA) with the MxPro QPCR software. The PCR conditions for CAR [CAR ex5 $\mathrm{S}$ and CAR ex6 AS] were as follows: after an initial denaturation at $95^{\circ} \mathrm{C}$ for 30 seconds, amplification was performed by denaturation at $94^{\circ} \mathrm{C}$ for 10 seconds followed by annealing/extension at $59^{\circ} \mathrm{C}$ for 10 seconds for 40 cycles. The PCR conditions for unspliced (CAR ex3 S and CAR int $3 \mathrm{AS}$ ) and spliced (CAR ex3 S and CAR ex4 AS) CAR were as follows: after an initial denaturation at $95^{\circ} \mathrm{C}$ for 30 seconds, amplification was performed by denaturation at $94^{\circ} \mathrm{C}$ for 10 seconds followed by annealing/extension at $60^{\circ} \mathrm{C}$ for 30 seconds for 40 cycles. The PCR conditions for CYP2B 6 were as follows: after an initial denaturation at $95^{\circ} \mathrm{C}$ for 30 seconds, amplification was performed by denaturation at $94^{\circ} \mathrm{C}$ for 10 seconds followed by annealing at $57^{\circ} \mathrm{C}$ for 10 seconds and extension $72^{\circ} \mathrm{C}$ for 20 seconds for 40 cycles. The PCR conditions for CYP3A4 were as follows: after an initial denaturation at $95^{\circ} \mathrm{C}$ for 30 seconds, amplification was performed by denaturation at $94^{\circ} \mathrm{C}$ for 10 seconds followed by annealing/extension at $60^{\circ} \mathrm{C}$ for 20 seconds for 40 cycles. The mRNA levels were normalized to those of GAPDH (Tsuchiya et al., 2004).

Reverse-Transcription PCR and Direct Sequence Analysis. The sequences of the primers used for the reverse-transcription PCR (RT-PCR) are shown in Table 1 . The region, including exon 3, intron 3 , and exon 4, was amplified by PCR using cDNA from HepG2 cells. The used primers were CAR ex $3 \mathrm{~S}$ and CAR ex4 AS, shown in Table 1. The PCR mixture consisted of cDNA, $1 \times$ PCR buffer, 10 pmol of each primer, and $0.5 \mathrm{U}$ of Gflex polymerase (Takara) in a final volume of $25 \mu$ l. After an initial denaturation at $94^{\circ} \mathrm{C}$ for 1 minute, amplification was performed with denaturation at $98^{\circ} \mathrm{C}$ for 10 seconds, annealing at $57^{\circ} \mathrm{C}$ for 15 seconds, and extension at $72^{\circ} \mathrm{C}$ for 90 seconds for 32 cycles followed by a final extension at $72^{\circ} \mathrm{C}$ for 5 minutes. The PCR products were subjected to electrophoresis using a $1.5 \%$ agarose gel. The specific products were purified and subjected to direct sequencing.

SDS-PAGE and Western Blot Analysis. For analysis of CAR, ADAR1, and GAPDH protein levels, cell homogenates from HepG2 cells were separated by $10 \%, 7.5 \%$, and $10 \%$ SDS-PAGE, respectively, and transferred to an Immobilon-P transfer membrane (Millipore, Billerica, MA). To analyze ADAR2 protein levels, the cell homogenates were separated by 7.5\% SDS-PAGE and transferred to a Protran nitrocellulose membrane (Whatman $\mathrm{GmbH}$, Dassel, Germany). The membranes were probed with each primary antibody and the corresponding fluorescent dye-conjugated secondary antibody. The band densities were quantified with an Odyssey Infrared Imaging system (LI-COR Biosciences). The CAR, ADAR1, and ADAR2 protein levels were normalized to GAPDH.

Assessment of the Stability of CAR mRNA. HepG2 cells were transfected with siRNA as described earlier. After 36 hours, the cells were treated with $10 \mu \mathrm{g} / \mathrm{ml} \alpha$-amanitin, a transcriptional inhibitor. Total RNA was prepared 0, 6, 12, and 24 hours later. The CAR mRNA level was evaluated by real-time RT-PCR as described earlier. 
TABLE 1

Primers for real-time reverse-transcription (RT) polymerase chain reaction

\begin{tabular}{ll}
\hline Primer & \multicolumn{1}{c}{ Sequence $\left(5^{\prime}-3^{\prime}\right)$} \\
\hline CAR ex5 S & CAG CTC ATC TGT TCA TCC AT \\
CAR ex6 AS & GGC TCC ATC TTC AAT TGT GT \\
CAR ex3 S & GTC AGC AAG ACT CAG AGG CG \\
CAR ex4 AS & CGT GTT TGA CAA GTT TGT AC \\
CAR int3 AS & CCT CTC GAT CTA GTA CTC TA \\
CYP2B6 RT S & CTT GCG GGG ATA TGG TGT GA \\
CYP2B6 RT AS & TCA AAC AGC TGG CCG AAT AC \\
CYP3A4 RT S ${ }^{a}$ & CCA AGC TAT GCT CTT CAC CG \\
CYP3A4 RT AS ${ }^{a}$ & TCA GGC TCC ACT TAC GGT GC \\
GAPDH RT S S & CCA GGG CTG CTT TTA ACT C \\
GAPDH RT AS ${ }^{b}$ & GCT CCC CCC TGC AAA TGA \\
\hline
\end{tabular}

AS, antisense; ex, exon; GAPDH, glyceraldehyde-3-phosphate dehydrogenase; int, intron; S, sense, .

${ }^{a}$ Takagi et al. (2008).

${ }^{b}$ Tsuchiya et al. (2004).

Reporter Plasmid Construction and Luciferase Assay. Reporter constructs containing the promoter region $(-2312$ to +58 or -317 to +1579 ) of CAR (Saito et al., 2010), termed pGL4.17/-2312+58 or pGL4.17/-317+1579, were generously provided by Drs. Kosuke Saito and Kaoru Kobayashi (Graduate School of Pharmaceutical Sciences, Chiba University). A reporter plasmid containing the 3'-UTR of CAR was constructed as follows. The 3 '-UTR of CAR $(+1251$ to +1379$)$ was amplified by PCR using HepG2 genomic DNA as a template and inserted into the pGL3p vector at the XbaI site downstream of the luciferase gene. The constructed luciferase reporter plasmid was termed pGL3p/3'-UTR.

HepG2 cells were seeded and $5 \mathrm{nM}$ siRNA was transfected as described earlier. After 24 hours, the cells were transfected with
A

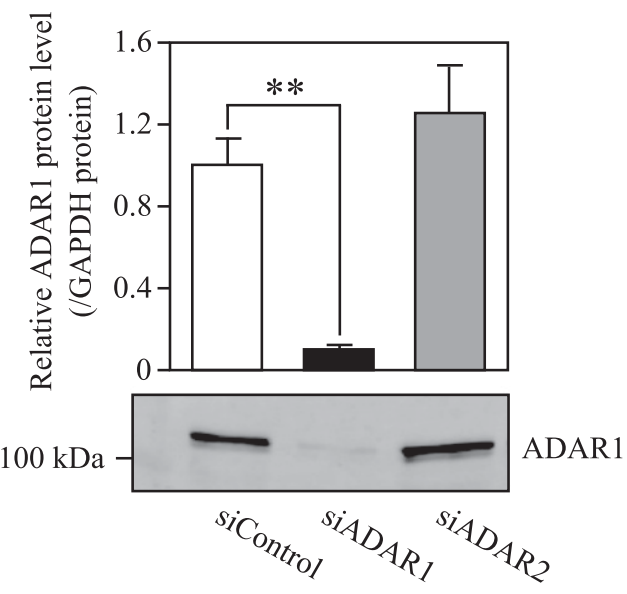

C

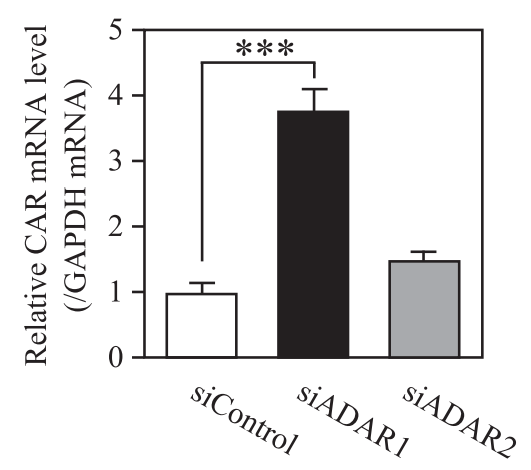

\section{B}

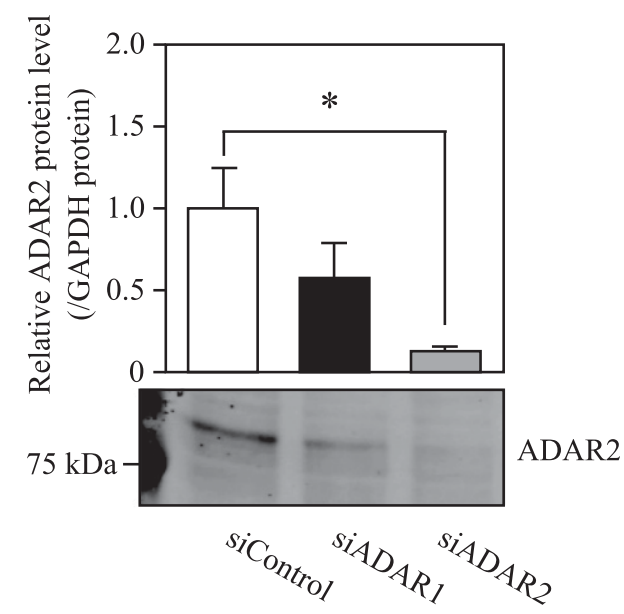

D

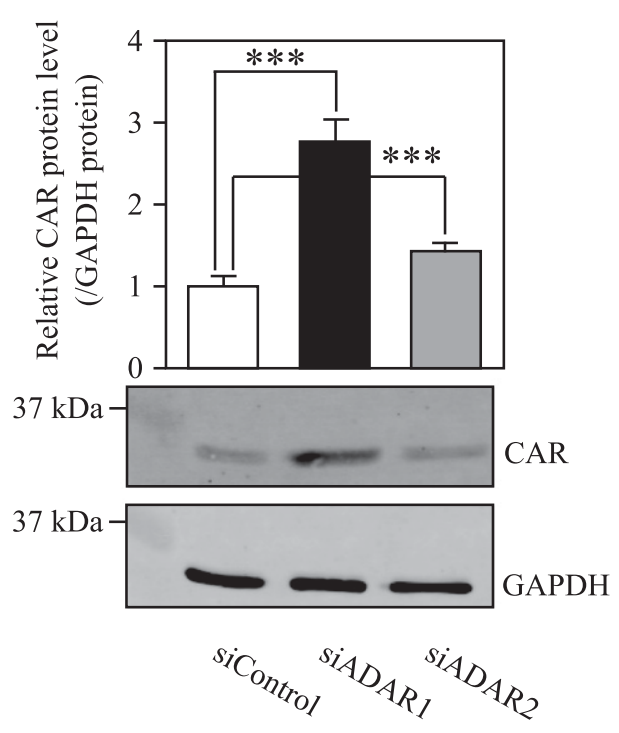

Fig. 1. Effects of ADAR1 or ADAR2 knockdown on CAR expression in HepG2 cells. ADAR1 (A), ADAR2 protein (B), CAR mRNA $(C)$, and CAR protein (D) in HepG2 cells 72 hours after transfection with $5 \mathrm{nM}$ siADAR1, siADAR2, or siControl were determined by Western blotting and were normalized to GAPDH. The values represent the levels relative to the siControl. Each column represents the mean \pm S.D. of three independent experiments. The values are expressed relative to the siControl. Each point represents the mean \pm S.D. $(n=3)$. $* P<0.05 ; * * P<0.01$; *** $P<0.001$. 
$200 \mathrm{ng}$ of pGL3 or pGL4.17 plasmid and $50 \mathrm{pg}$ of pNL1.1 plasmid. After incubation for 48 hours, luciferase activity was measured with a luminometer using the Nano-Glo Dual-Luciferase Reporter Assay System.

Evaluation of the Transactivity of CYP3A4 by Luciferase Assay. A reporter plasmid pCYP3A4-362-7.7K containing the promoter region $(-362$ to +11$)$ and distal enhancer region $(-7836$ to -7200) of the CYP3A4 gene was provided by Drs. Kiyoshi Nagata and Yasushi Yamazoe (Graduate School of Pharmaceutical Sciences, Tohoku University). HepG2 cells were seeded into 96-well plates, and $5 \mathrm{nM}$ siRNA was transfected with RNAiMAX. After 24 hours, $200 \mathrm{ng}$ of pCYP3A4-362-7.7K along with $50 \mathrm{pg}$ of pNL1.1 plasmid was transfected. After incubation for 24 hours, these cells were treated with $3 \mathrm{mM} \mathrm{PB}$ for 24 hours, and then the luciferase activity was measured. Firefly luciferase activity for each construct was normalized to NanoLuc luciferase activity.

Statistical Analyses. Statistical significance was determined by analysis of variance followed by Dunnett's multiple comparisons test or Tukey's method test. The comparison of two groups was made with an unpaired, two-tailed Student's $t$ test. A value of $P<0.05$ was considered to be statistically significant.

\section{Results}

Knockdown of ADARs Results in Increases in CAR Expression in HepG2 Cells. To investigate whether ADAR1 and ADAR2 affect human CAR expression, their expressions in HepG2 cells were knocked down using siRNA. As shown in Fig. 1, A and B, ADAR1 and ADAR2 protein levels were significantly decreased by transfection of siADAR 1 and siADAR2, respectively. The knockdown of ADAR1 resulted in significant increases in CAR mRNA (Fig. 1C) and protein (Fig. 1D) levels. The knockdown of ADAR2 slightly but significantly increased CAR protein levels (Fig. 1D). These results suggest that ADARs negatively regulate CAR expression.

Knockdown of ADARs Augments the Induction of CYP2B6 and CYP3A4. CAR is activated by some synthetic compounds, drugs, or natural products (Chang and Waxman, 2006) and transactivates target gene expression. We investigated whether the siADAR-mediated upregulation of CAR affects the induction of its downstream CYP3A4 and CYP2B6. First, the effects of knockdown of ADARs on the transactivity of CYP3A4 was evaluated through a luciferase assay using a reporter plasmid containing the CYP3A4 promoter and enhancer regions to which CAR binds (Schuetz et al., 1998; Takada et al., 2004) (pCYP3A4-362-7.7K). Treatment with PB, an activator of CAR, resulted in a significant increase in luciferase activity of pCYP3A4-362-7.7K plasmid in siADAR1transfected cells but not in siControl- or siADAR2-transfected cells, suggesting that the increase in CAR expression by ADAR1 knockdown enhanced PB-mediated CYP3A4 induction (Fig. 2A).
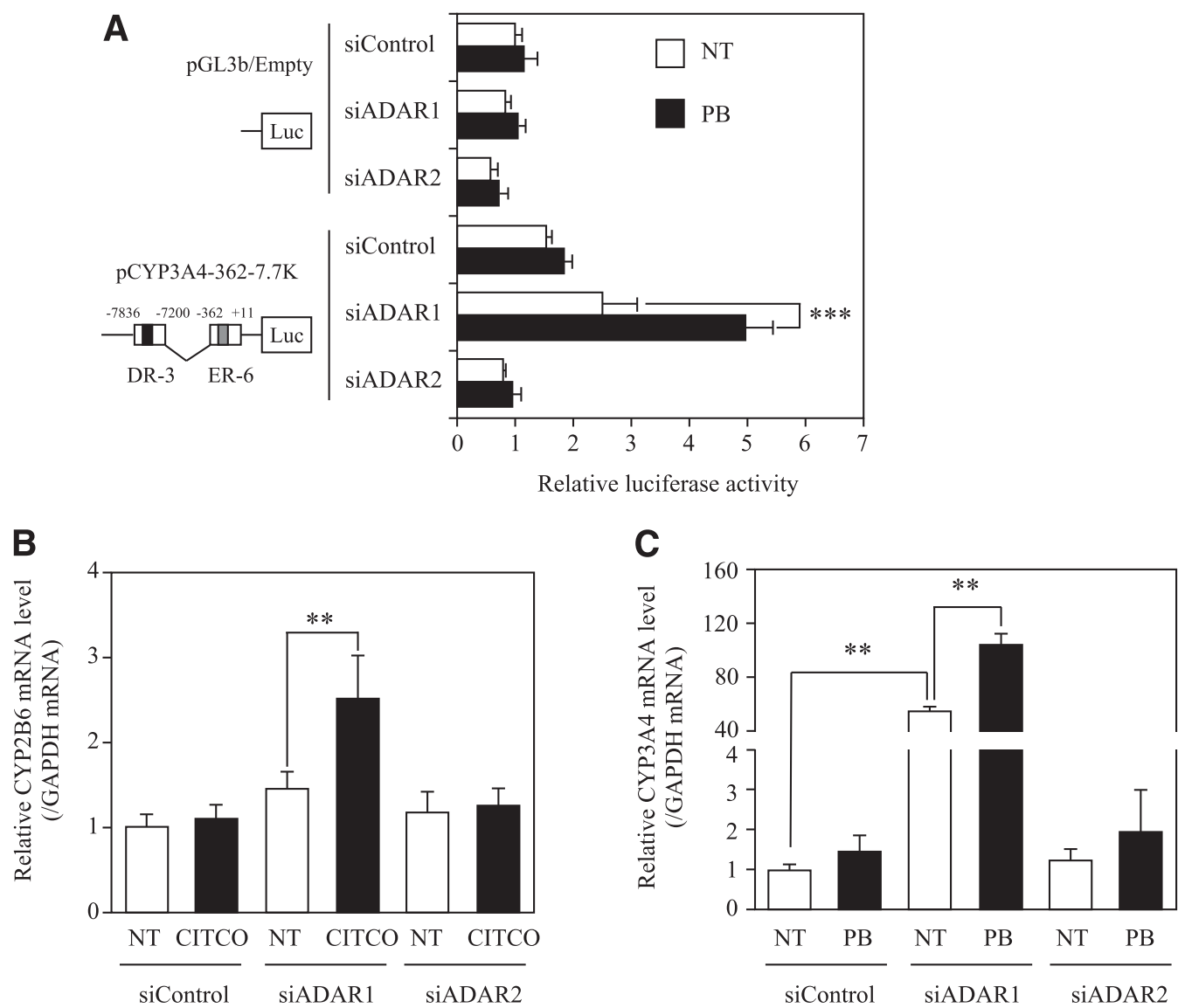

Fig. 2. Effects of ADAR1 or ADAR2 knockdown on induction of CYP2B6 and CYP3A4. (A) HepG2 cells were transfected with siADAR1 or siADAR2. After 24 hours, the cells were transfected with pCYP3A4-362-7.7K along with pNL1.1 and siADAR1 or siADAR2. Firefly luciferase activity was normalized to NanoLuc luciferase activity. The values are expressed relative to the pGL3b plasmid. (B and C) After transfection of siADAR1 or siADAR2, the HepG2 cells were treated with $100 \mathrm{nM}$ CITCO (B) or $1 \mathrm{mM} \mathrm{PB} \mathrm{(C).} \mathrm{After} 24$ hours, CYP2B6 (B) and CYP3A4 (C) mRNA levels were determined by real-time RT-PCR and were normalized to GAPDH. The values are expressed relative to the control. Each column represents the mean \pm S.D. of three independent experiments. ${ }^{* *} P<0.01 ; * * * P<0.001$. NT, No treatment. 
In addition, treatment with CITCO, a ligand of CAR, increased endogenous CYP2B6 mRNA levels in siADAR1-transfected cells but not in siControl- or siADAR2-transfected cells (Fig. 2B). Moreover, CYP3A4 mRNA levels were markedly increased by ADAR1 knockdown, and PB-mediated induction of CYP3A4 mRNA was observed in siADAR1-transfected cells (Fig. 2C). Collectively, these data demonstrated that the upregulation of CAR by knockdown of ADAR1 results in the enhanced induction of its downstream genes.

Knockdown of ADARs Does Not Affect Transactivity of CAR. To examine whether the increase in CAR levels by knockdown of ADARs is attributed to the accelerated transactivation, we performed luciferase assays using the reporter plasmid containing the promoter region of CAR $(-2312$ to +58 or -317 to +1579 ). The luciferase activities of pGL4.17/$2312+58$ and pGL4.17/-317+1579 were not changed by transfection of siADARs (Fig. 3), indicating that ADARs do not affect the transactivity of CAR.

Knockdown of ADARs Attenuates Degradation of CAR mRNA. To examine whether the increase in CAR mRNA by knockdown of ADAR1 is due to the decreased mRNA degradation, the stability of CAR mRNA was evaluated. In the siControl-transfected cells, CAR mRNA level was gradually decreased by treatment with $\alpha$-amanitin, a transcriptional inhibitor (Fig. 4A). The half-life was calculated to be 6.6 hours. In the siADAR1-transfected cells, CAR mRNA was relatively stable with a half-life of 18.6 hours. These results indicate that the increase in CAR expression by ADAR1 knockdown would be due to the increased stability of CAR mRNA.

To investigate the involvement of the $3^{\prime}$-UTR in ADAR1mediated regulation of CAR mRNA stability, luciferase assays using a reporter plasmid containing the $3^{\prime}$-UTR of CAR were conducted. The luciferase activity of the pGL3p/3'-UTR plasmid was increased by knockdown of ADARs (Fig. 4B), but similar results were obtained in the case of the pGL3p/ empty plasmid. The results suggest that the 3 '-UTR is not relevant to the increased stability of CAR mRNA by knockdown of ADAR1.

Knockdown of ADARs Enhances the Splicing of CAR. REDIportal (http://srv00.recas.ba.infn.it/atlas/), a database of RNA-editing sites, indicates that there are
22 RNA-edited sites in intron 3 of CAR. We examined whether ADARs affect the splicing event at intron 3 of CAR. To examine what kinds of transcripts are produced by the splicing of this intron, RT-PCR was performed using CAR ex3 $\mathrm{S}$ and CAR ex4 AS primers, shown in Fig. 5A. As shown in Fig. 5B, two PCR products were observed in siControltransfected cells. Direct sequence analysis revealed that the longer (2746 bp) and shorter (233 bp) products corresponded to the unspliced and spliced transcripts, respectively. By the analysis of the sequence of the unspliced transcript, we noticed that there are no RNA-edited sites in the intron 3 of CAR, because overlapped peaks of "G" to "A" were not observed, as shown in a typical sequencing electropherogram (Fig. 5C), even though this region includes RNA-edited sites registered in REDIportal (shown by vertical arrows). The band intensities of the unspliced and spliced transcripts were decreased and increased by knockdown of ADAR1, respectively. Since RTPCR lacks quantitativeness, real-time RT-PCR analyses, in which unspliced transcript and spliced transcript would be specifically amplified, were performed for quantitative evaluation. CAR ex3 and CAR int 3 AS primers were used to evaluate the unspliced transcript. CAR ex3 and CAR ex4 AS primers were used to amplify only the spliced transcript, and the extension time was set to be shorter than that in RT-PCR, as described in detail in the Materials and Methods section. As shown in Fig. 5D, the level of the unspliced CAR transcript tended to be decreased by knockdown of ADARs, whereas that of the spliced CAR transcript was significantly increased by knockdown of ADARs. These results indicated that ADARs attenuate the splicing of CAR intron 3 in an RNA editingindependent manner, possibly resulting in downregulation of CAR expression.

\section{Discussion}

The expression of CAR is regulated at the transcriptional level by transcription factors, such as glucocorticoid receptor (Pascussi et al., 2003), hepatocyte nuclear factor $4 \alpha$ (Ding et al., 2006), aryl hydrocarbon receptor (Patel et al., 2007), proliferator-activated receptor $\alpha$ (Wieneke et al., 2007), and retinoic acid receptor (Saito et al., 2010). The present study demonstrated a new post-transcriptional regulatory mechanism:

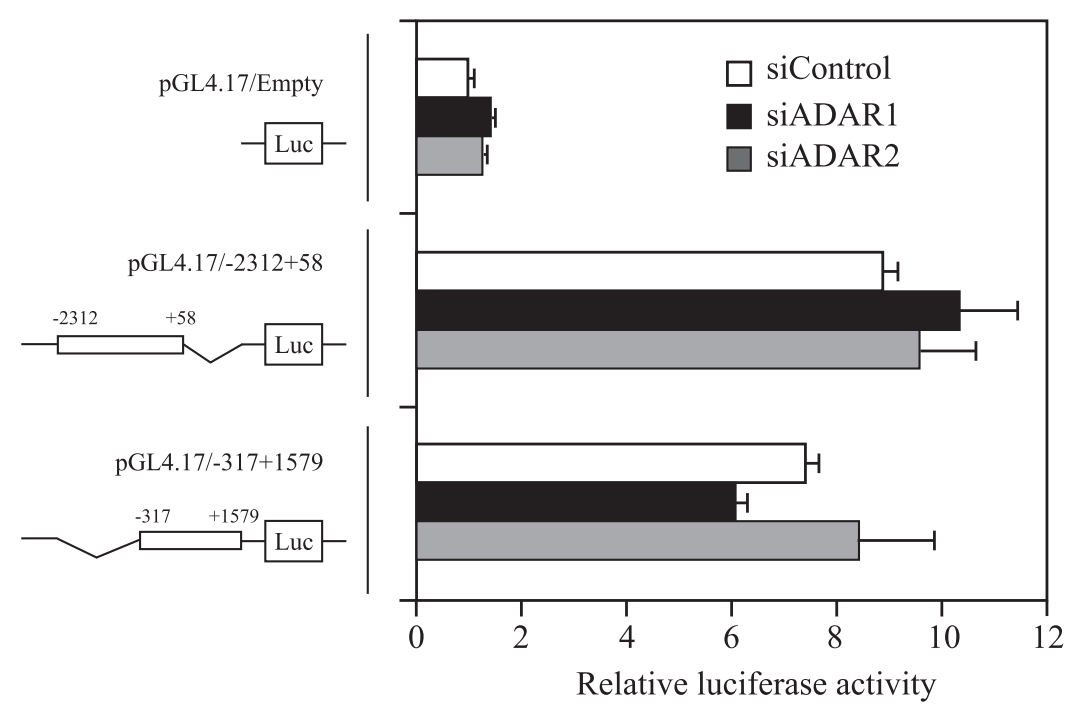

Fig. 3. Effects of ADAR1 or ADAR2 knockdown on transactivity of CAR. HepG2 cells were transfected with siADAR1 or siADAR2. After 24 hours, the cells were transfected with $200 \mathrm{ng}$ of the reporter plasmid containing the promoter region of CAR along with $50 \mathrm{pg}$ of the pNL1.1 plasmid. Firefly luciferase activity was normalized to NanoLuc luciferase activity. The values are expressed relative to the pGL4.17 plasmid and siControl. Each column represents the mean \pm S.D. of three independent experiments. 
A

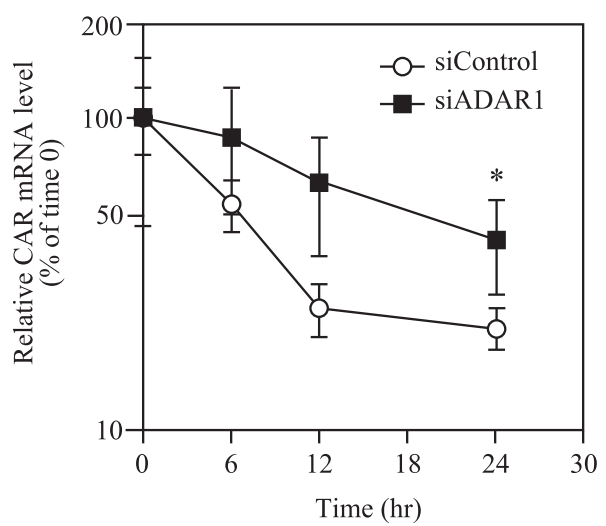

B

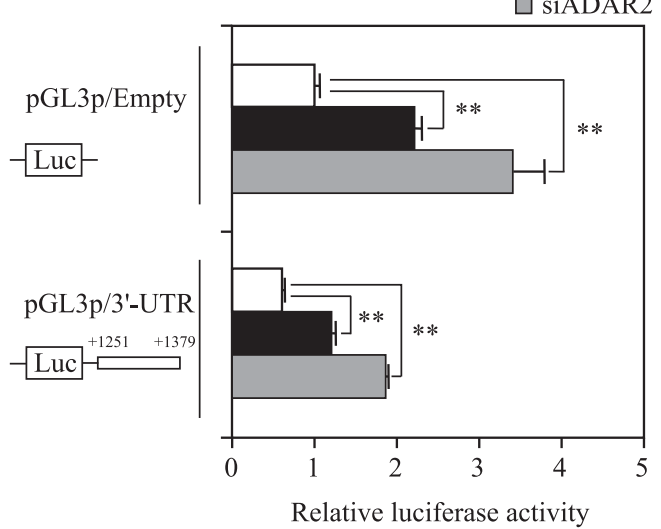

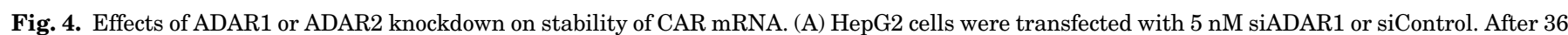

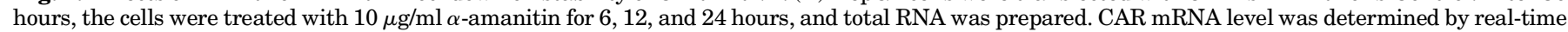

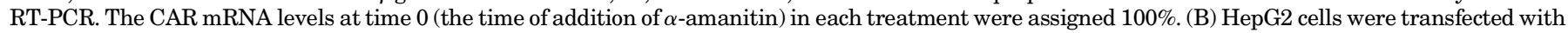

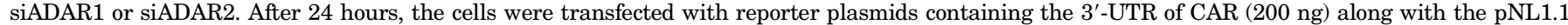

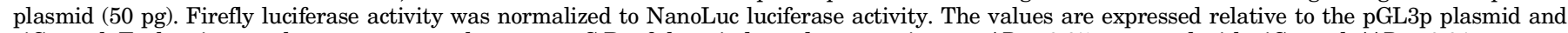
siControl. Each point or column represents the mean \pm S.D. of three independent experiments. ${ }^{*} P<0.05$ compared with siControl; ${ }^{*} * P<0.01$.

ADAR1 negatively regulates the expression of CAR by attenuating splicing.

Splicing is a process to remove introns from pre-mRNA and ligate adjacent exons to form mature mRNA. Most introns are flanked by $5^{\prime}$-GT-AG-3' splice sites, and RNA editing in introns has the potential to affect splicing (Samuel, 2019). The first example of RNA editing-dependent alternative splicing during the processing of pre-mRNA was ADAR2mediated autoediting of its own pre-mRNA. This autoediting creates a 3'AG splice site (AA changed to $\mathrm{AI}$ ), leading to alternative splicing (Dawson et al., 2004). Recently, it was reported that intronic editing of the heterogeneous nuclear ribonucleoprotein L-like transcript by ADAR1 and ADAR2 generates another variant containing an additional exon (Chen et al., 2018). We noticed that 22 RNA-edited sites are registered within the two inverted Alu elements, which can form dsRNA structures, in intron 3 of CAR in REDIportal. We found that the knockdown of ADARs enhances the efficiency of splicing at intron 3 (Fig. 5). We examined whether intron 3 of CAR pre-mRNA in HepG2 cells was actually subjected to RNA editing by direct sequence analysis using the longer PCR product (2746 bp) in Fig. 5B. As a result, no editing sites were observed in the two Alu elements located in intron 3 of CAR (the typical result is shown in Fig. 5C). Recently, growing evidence suggests that ADARs have potential to regulate gene expression in an RNA editing-independent manner. A study reported that overexpression of active (wild-type) or inactive (by an amino acid change, E397A) ADAR2 causes aberrant splicing of its own pre-mRNA, suggesting an RNA editing-independent role of ADAR2 in splicing (Agranat et al., 2010). In addition, ADAR1 competitively inhibits binding of staufen 1 to the dsRNA in the 3 '-UTR of cyclin G1 or cancer susceptibility candidate 5 to antagonize Staufen1-mediated mRNA decay in an RNA editing-independent manner (Sakurai et al., 2017). The editing-independent function would require only dsRNA binding domains but not the deaminase domain of ADAR proteins. Referring to these examples, it can be assumed that ADAR1 may bind to the dsRNA structure in intron 3 of CAR pre-mRNA, leading to the inhibition of splicing.
Aberrant splicing products are degraded by nonsensemediated mRNA decay, a mechanism that eliminates mRNAs containing premature translation-termination codons (PTCs) (McGlincy and Smith, 2008). We found that knockdown of ADAR1 attenuated the degradation of CAR mRNA through enhancing the efficiency of splicing (Fig. 4A). We noticed that the retention of intron 3 in CAR mRNA results in the creation of PTCs (data not shown). Collectively, ADAR1 causes aberrant splicing of CAR pre-mRNA to create PTC, possibly leading to the degradation of the aberrant transcript by nonsense-mediated mRNA decay.

Recently, we reported that ADARs regulate the $\mathrm{P} 450$ isoform in HepaRG cells (Nozaki et al., 2019). In that study, an increase in CYP3A4 expression by knockdown of ADAR1 was observed. CYP3A4 is a target gene (Schuetz et al., 1998; Takada et al., 2004) of CAR. The present study demonstrated that the knockdown of ADAR1 resulted in enhanced PB-mediated transactivation of CYP3A4 (Fig. 2A) and increased levels of endogenous CYP3A4 mRNA (Fig. 2C). Therefore, the upregulation of CAR by knockdown of ADAR1 would be one of the underlying mechanisms of the increased CYP3A4 expression by knockdown of ADAR1. In addition to CYP3A4, CYP2B6 transcription is also regulated by CAR (Hedrich et al., 2016). There are large interindividual differences in CYP3A4 (50-fold) and CYP2B6 (158-fold) expressions in human livers (Ozdemir et al., 2000; Rodríguez-Antona et al., 2001). Our previous study revealed a large interindividual variation (220-fold) in hepatic ADAR1 protein levels (Nakano et al., 2016). Therefore, it is possible that the variation in ADAR1 expression could be one of the factors causing the interindividual variation in such CAR-regulated P450 expression. It remains unknown what factors cause the interindividual difference in hepatic ADAR1 expression. It is worth investigating whether xenobiotics such as drugs, herbal products, and environmental pollutants or genetic polymorphisms affect $\mathrm{ADAR}$ expression or function. Such knowledge would enhance the significance of ADARmediated post-transcriptional regulation in drug metabolism.

CAR regulates not only drug-metabolizing enzymes but also energy metabolism. It has been reported that activation of CAR leads to amelioration of some metabolic diseases, such as 
A

CAR pre-mRNA

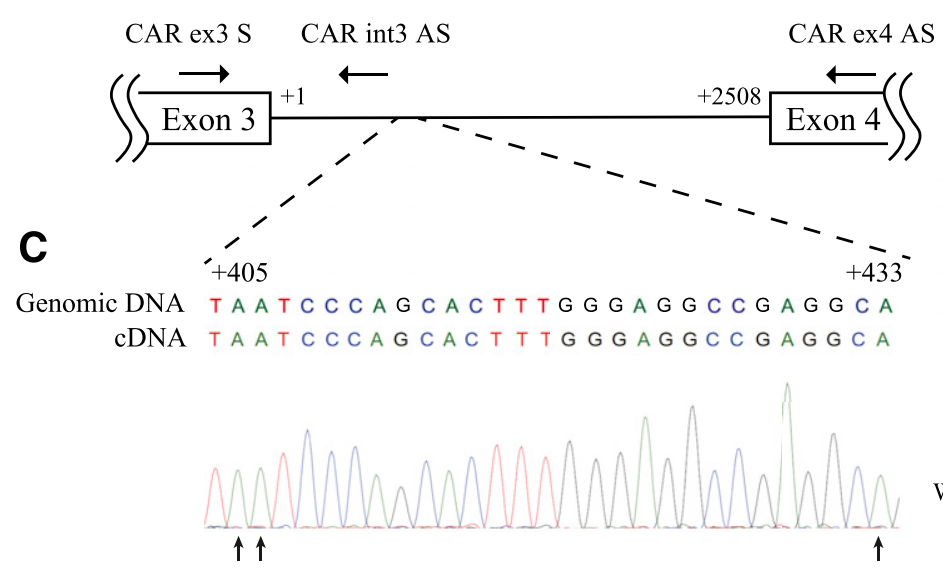

B

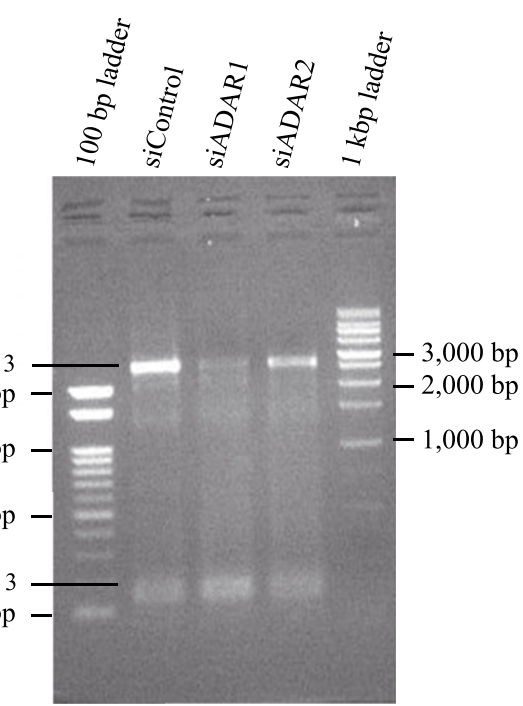

D

CAR ex3 S CAR int3 AS

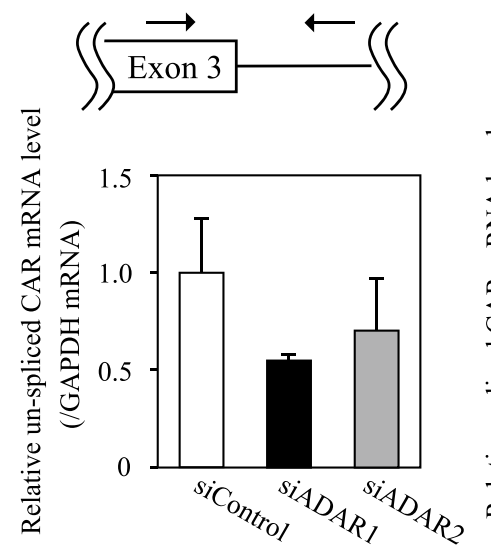

CAR ex3 S CAR ex4 AS
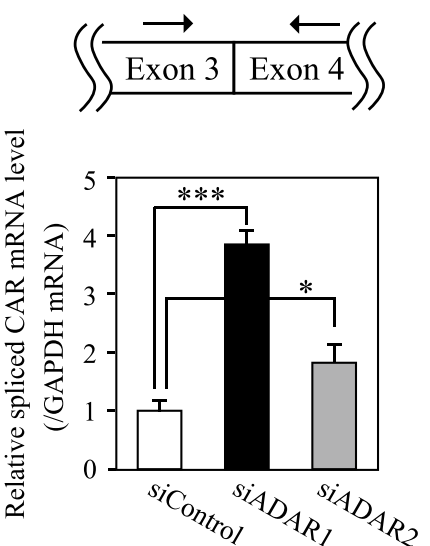

Fig. 5. Effects of ADAR1 or ADAR2 knockdown on splicing of intron 3 of CAR. (A) Gene structure from exon3 to exon 4 of human CAR pre-mRNA is schematically represented. Open rectangles represent exons. The nucleotide numbering refers to the $5^{\prime}$ end of intron 3 as 1 . Arrows represent primers used in (B) and (C). (B) Electropherogram of the PCR amplicon by using CAR ex3 S and CAR ex4 AS primers and cDNA from HepG2 cells. The PCR products of 2746 and $233 \mathrm{bp}$ correspond to unspliced and spliced transcripts, respectively. (C) A representative electropherogram from direct sequencing of the PCR product derived from the unspliced transcript (B). The nucleotide numbering refers to the $5^{\prime}$ end of intron 3 as 1 . Vertical arrows show the RNA editing sites registered in the REDIportal. (D) The levels of unspliced and spliced transcripts of CAR were evaluated by real-time RT-PCR using primers depicted by arrows. The values are expressed relative to the control. Each column represents the mean \pm S.D. of three independent experiments. $* P<0.05 ; * * * P<0.001$. int3, intron 3 .

atherosclerosis (Sberna et al., 2011), obesity (Gao et al., 2009), fatty liver disease (Dong et al., 2009), and diabetes (Dong et al., 2009). In addition, CAR is involved in the exacerbation of hepatic fibrosis in a mouse dietary model of nonalcoholic steatohepatitis (Yamazaki et al., 2007). A recent study reported that hepatocyte-specific ADAR1 knockout mice display massive liver damage with fibrogenesis, suggesting that ADAR1 restrains the development of hepatic fibrosis (BenShoshan et al., 2017). Combined with the present findings that ADAR1 negatively regulates CAR expression, a possibility is surmised that ADAR1 has a role in protecting the liver from fibrosis by repressing CAR expression. It is worth investigating such a possibility in future studies. It is of interest to examine whether the interindividual difference in ADAR1 expression may cause interindividual variability in the CARregulating hepatic function.

In conclusion, we found that ADAR 1 downregulates CAR expression by repressing splicing of CAR and facilitating its mRNA degradation. ADAR1 may have a role in control of liver function, including drug metabolism and energy metabolism, via regulation of CAR. The present study provided new insights into ADAR-mediated gene regulation and the potential biologic significance of hepatic ADAR1.

\section{Acknowledgments}

The authors thank Dr. Kiyoshi Nagata and Dr. Yasushi Yamazoe (Graduate School of Pharmaceutical Sciences, Tohoku University, Sendai, Japan) for providing pCYP3A4-362-7.7K plasmid and Dr. Kosuke Saito and Dr. Kaoru Kobayashi (School of Pharmaceutical Sciences, Chiba University) for providing pGL4.17/-2312+58 and pGL4.17/-317+1579.

\section{Authorship Contributions}

Participated in research design: Nakano, Fukami, Nakajima.

Conducted experiments: Nakano.

Contributed new reagents or analytic tools: Nakano.

Performed data analysis: Nakano, Nakajima.

Wrote or contributed to the writing of the manuscript: Nakano, Nakajima. 


\section{References}

Agranat L, Sperling J, and Sperling R (2010) A novel tissue-specific alternatively spliced form of the A-to-I RNA editing enzyme ADAR2. RNA Biol 7:253-262.

Ben-Shoshan SO, Kagan P, Sultan M, Barabash Z, Dor C, Jacob-Hirsch J, Harmelin A, Pappo O, Marcu-Malina V, Ben-Ari Z, et al. (2017) ADAR1 deletion induces $\mathrm{NF}_{\kappa} \mathrm{B}$ and interferon signaling dependent liver inflammation and fibrosis. RNA Biol 14:587-602.

Chang TKH and Waxman DJ (2006) Synthetic drugs and natural products as modulators of constitutive androstane receptor (CAR) and pregnane X receptor (PXR). Drug Metab Rev 38:51-73.

Chen Y-T, Chang IY-F, Liu H, Ma C-P, Kuo Y-P, Shih C-T, Shih Y-H, Kang L, and Tan BC-M (2018) Tumor-associated intronic editing of HNRPLL generates a novel splicing variant linked to cell proliferation. J Biol Chem 293:10158-10171.

Dawson TR, Sansam CL, and Emeson RB (2004) Structure and sequence determinants required for the RNA editing of ADAR2 substrates. J Biol Chem 279: 4941-4951.

Ding X, Lichti K, Kim I, Gonzalez FJ, and Staudinger JL (2006) Regulation of constitutive androstane receptor and its target genes by fasting cAMP, hepatocyte nuclear factor alpha, and the coactivator peroxisome proliferator-activated receptor gamma coactivator-1alpha. J Biol Chem 281:26540-26551.

Dong B, Saha PK, Huang W, Chen W, Abu-Elheiga LA, Wakil SJ, Stevens RD, Ilkayeva O, Newgard CB, Chan L, et al. (2009) Activation of nuclear receptor CAR ameliorates diabetes and fatty liver disease. Proc Natl Acad Sci USA 106: 18831-18836.

Gao J, He J, Zhai Y, Wada T, and Xie W (2009) The constitutive androstane receptor is an anti-obesity nuclear receptor that improves insulin sensitivity. J Biol Chem 284:25984-25992.

Gerber A, O'Connell MA, and Keller W (1997) Two forms of human double-stranded RNA-specific editase 1 (hRED1) generated by the insertion of an Alu cassette. RNA 3:453-463.

Hedrich WD, Hassan HE, and Wang H (2016) Insights into CYP2B6-mediated drugdrug interactions. Acta Pharm Sin B 6:413-425.

Kim U, Wang Y, Sanford T, Zeng Y, and Nishikura K (1994) Molecular cloning of cDNA for double-stranded RNA adenosine deaminase, a candidate enzyme for nuclear RNA editing. Proc Natl Acad Sci USA 91:11457-11461.

McGlincy NJ and Smith CWJ (2008) Alternative splicing resulting in nonsensemediated mRNA decay: what is the meaning of nonsense? Trends Biochem Sci 33: 385-393.

Moore DD, Kato S, Xie W, Mangelsdorf DJ, Schmidt DR, Xiao R, and Kliewer SA (2006) International Union of Pharmacology. LXII. The NR1H and NR1I receptors: constitutive androstane receptor, pregnene $\mathrm{X}$ receptor, farnesoid $\mathrm{X}$ receptor alpha, farnesoid X receptor beta, liver X receptor alpha, liver X receptor beta, and vitamin D receptor. Pharmacol Rev 58:742-759.

Nakano M, Fukami T, Gotoh S, Takamiya M, Aoki Y, and Nakajima M (2016) RNA editing modulates human hepatic aryl hydrocarbon receptor expression by creating microRNA recognition sequence. J Biol Chem 291:894-903.

Nakano M and Nakajima M (2018) Significance of A-to-I RNA editing of transcripts modulating pharmacokinetics and pharmacodynamics. Pharmacol Ther 181: $13-21$.

Negishi M and Honkakoski P (2000) Induction of drug metabolism by nuclear receptor CAR: molecular mechanisms and implications for drug research. Eur $J$ Pharm Sci 11:259-264.

Nishikura K (2016) A-to-I editing of coding and non-coding RNAs by ADARs. Nat Rev Mol Cell Biol 17:83-96.

Nozaki K, Nakano M, Iwakami C, Fukami T, and Nakajima M (2019) RNA editing enzymes modulate the expression of hepatic CYP2B6, CYP2C8, and other cytochrome P450 isoforms. Drug Metab Dispos 47:639-647.

Ozdemir V, Kalow W, Tang BK, Paterson AD, Walker SE, Endrenyi L, and Kashuba $\mathrm{AD}$ (2000) Evaluation of the genetic component of variability in CYP3A4 activity: a repeated drug administration method. Pharmacogenetics 10:373-388.
Pascussi JM, Busson-Le Coniat M, Maurel P, and Vilarem M-J (2003) Transcriptional analysis of the orphan nuclear receptor constitutive androstane receptor (NR1I3) gene promoter: identification of a distal glucocorticoid response element. Mol Endocrinol 17:42-55.

Patel RD, Hollingshead BD, Omiecinski CJ, and Perdew GH (2007) Aryl-hydrocarbon receptor activation regulates constitutive androstane receptor levels in murine and human liver. Hepatology 46:209-218.

Peng Z, Cheng Y, Tan BC, Kang L, Tian Z, Zhu Y, Zhang W, Liang Y, Hu X, Tan X, et al. (2012) Comprehensive analysis of RNA-Seq data reveals extensive RNA editing in a human transcriptome. Nat Biotechnol 30:253-260.

Picardi E, D'Erchia AM, Lo Giudice C, and Pesole G (2017) REDIportal: a comprehensive database of A-to-I RNA editing events in humans. Nucleic Acids Res $\mathbf{4 5}$ (D1):D750-D757.

Rodríguez-Antona C, Donato MT, Pareja E, Gómez-Lechón MJ, and Castell JV (2001) Cytochrome P-450 mRNA expression in human liver and its relationship with enzyme activity. Arch Biochem Biophys 393:308-315.

Saito K, Kobayashi K, Mizuno Y, Furihata T, and Chiba K (2010) Constitutive androstane/active receptor is a target of retinoic acid receptor in humans. Biochem Pharmacol 80:129-135.

Sakurai M, Shiromoto Y, Ota H, Song C, Kossenkov AV, Wickramasinghe J, Showe LC, Skordalakes E, Tang H-Y, Speicher DW, et al. (2017) ADAR1 controls apoptosis of stressed cells by inhibiting Staufen1-mediated mRNA decay. Nat Struct Mol Biol 24:534-543.

Samuel CE (2019) Adenosine deaminase acting on RNA (ADAR1), a suppressor of double-stranded RNA-triggered innate immune responses. $J$ Biol Chem 294: $1710-1720$

Sberna AL, Assem M, Gautier T, Grober J, Guiu B, Jeannin A, Pais de Barros J-P, Athias A, Lagrost L, and Masson D (2011) Constitutive androstane receptor activation stimulates faecal bile acid excretion and reverse cholesterol transport in mice. J Hepatol 55:154-161.

Schuetz EG, Brimer C, and Schuetz JD (1998) Environmental xenobiotics and the antihormones cyproterone acetate and spironolactone use the nuclear hormone pregnenolone X receptor to activate the CYP3A23 hormone response element. Mol Pharmacol 54:1113-1117.

Slotkin W and Nishikura K (2013) Adenosine-to-inosine RNA editing and human disease. Genome Med 5:105.

Takada T, Ogino M, Miyata M, Shimada M, Nagata K, and Yamazoe Y (2004) Differences in transactivation between rat CYP3A1 and human CYP3A4 genes by human pregnane X receptor. Drug Metab Pharmacokinet 19:103-113.

Takagi S, Nakajima M, Mohri T, and Yokoi T (2008) Post-transcriptional regulation of human pregnane $\mathrm{X}$ receptor by micro-RNA affects the expression of cytochrome P450 3A4. J Biol Chem 283:9674-9680.

Tsuchiya Y, Nakajima M, Kyo S, Kanaya T, Inoue M, and Yokoi T (2004) Human CYP1B1 is regulated by estradiol via estrogen receptor. Cancer Res 64:3119-3125. Wang Y and Liang H (2018) When MicroRNAs meet RNA editing in cancer: a nucleotide change can make a difference. BioEssays 40.

Wieneke N, Hirsch-Ernst KI, Kuna M, Kersten S, and Püschel GP (2007) PPARalpha-dependent induction of the energy homeostasis-regulating nuclear receptor NR1i3 (CAR) in rat hepatocytes: potential role in starvation adaptation. FEBS Lett 581:5617-5626.

Yamazaki Y, Kakizaki S, Horiguchi N, Sohara N, Sato K, Takagi H, Mori M, and Negishi M (2007) The role of the nuclear receptor constitutive androstane receptor in the pathogenesis of non-alcoholic steatohepatitis. Gut 56:565-574.

Address correspondence to: Dr. Miki Nakajima, Drug Metabolism and Toxicology, Faculty of Pharmaceutical Sciences, Kanazawa University Kakuma-machi, Kanazawa 920-1192, Japan. E-mail: nmiki@p.kanazawau.ac.jp 